\section{Qualidade e eqüidade da atenção ao pré-natal e ao parto em Criciúma, Santa Catarina, Sul do Brasil}

\section{Quality and equity in antenatal care and during delivery in Criciúma, Santa Catarina, in Southern Brazil}

\author{
Nelson A. Neumann \\ Pastoral da Criança \\ Conferência Nacional dos Bispos do Brasil (CNBB) \\ Rua Jacarezinho, 1691 \\ 80810-900 - Curitiba, PR \\ nneumann@rebidia.org.br
}

\section{Oswaldo Y. Tanaka}

Departamento de Prática de Saúde Pública

Faculdade de Saúde Pública

Universidade de São Paulo

\section{Cesar G. Victora}

Departamento de Medicina Social

Faculdade de Medicina

Universidade Federal de Pelotas

\section{Resumo}

Este estudo transversal de base populacional teve por objetivo avaliar a qualidade e a eqüidade da atenção ao pré-natal, ao parto e ao recém-nascido em uma amostra probabilística de 2180 crianças menores de três anos residentes na área urbana do município de Criciúma, SC. 96,6\% das mães destas crianças realizaram pelo menos uma consulta de pré-natal; o parto hospitalar foi praticamente universal. As mães de menor renda iniciaram o pré-natal mais tarde e realizaram menos consultas. Gestantes com maior risco reprodutivo apresentaram menor percentagem de atendimento com qualidade adequada. Dos 13 procedimentos de pré-natal e parto pesquisados, $01^{\circ} \mathrm{e} 4^{\circ}$ quartil de renda familiar realizaram 8,6 e 9,4 procedimentos, respectivamente. Os exames ginecológico especular e de mamas foram os menos realizados (51\% para ambos). As gestantes do $4^{\circ}$ quartil de renda familiar total tiveram 1,5 e 1,9 vezes mais estes procedimentos em relação às do $1^{\circ}$ quartil. Esforços devem ser feitos no sentido de captar precocemente as mães provenientes de famílias de menor poder aquisitivo e deve ser utilizado o enfoque de risco com discriminação positiva. Os médicos devem ser re-treinados quanto aos seus procedimentos tanto no pré-natal quanto na assistência ao parto.

Palavras-chave: Criança. Gestante. Eqüidade. Recém-nascido. Atenção primária em saúde. Pré-natal. Assistência ao parto. Avaliação de serviços de saúde.

Juraci A. Cesar

Divisão de Epidemiologia

Hospital Dr. Miguel Riet Corrêa Jr.

Departamento Materno-Infantil

Universidade Federal do Rio Grande 
Abstract

This population-based cross-sectional study was carried out with a sample of 2,180 children less than three years old living in the urban area of Criciúma, Santa Catarina State, in Southern Brazil. The study aimed to evaluate equity issues related to the quality of antenatal care and newborn care during delivery. $96.6 \%$ of mothers had at least one medical check-up during antenatal care. Almost all deliveries took place in a hospital. Lowincome mothers began antenatal care later and had less medical check-ups. Pregnant women with higher reproductive risk scores received less appropriate care in the period. Among 13 recommended procedures for antenatal care and during delivery, mothers belonging to the first and quarter quartiles received 8.6 and 9.4 of the recommended procedures, respectively. Pelvic and breast examinations were the least frequently performed of all recommended procedures (51\%). Women in the highest income quartile were 1.5 times more likely to have a pelvic exam, and 1.9 times more likely to have their breasts examined, than those in the lowest quartile. Health services should focus on the poorest and highest risk pregnant women. Doctors and health workers should be trained and motivated toward this purpose.

Key Words: Child. Pregnant women. Equity. Primary health care. Evaluation of services. Antenatal care. Health service evaluation.

\section{Introdução}

Os serviços de saúde são regidos pela "lei dos cuidados inversos", fenômeno praticamente universal, descrito por Hart ${ }^{1}$ há três décadas: os que mais necessitam de cuidados em saúde são os menos por eles beneficiados. Assim, os programas de saúde atingem maiores coberturas nos grupos populacionais que deles menos necessitam ${ }^{2}$. Em amplos estudos realizados nas regiões Sul, Sudeste e Nordeste do Brasil, ficou evidente que a cobertura de ações básicas de saúde materno-infantil é sistematicamente superior nos níveis socioeconômicos mais eleva$\operatorname{dos}^{3-7}$.

A gravidez e o parto são determinantes importantes do estado de saúde da mulher. A gravidez pode ser, ainda, o único contato que uma mulher em idade reprodutiva tem com os serviços de saúde. Trata-se, portanto, de valiosa oportunidade para intervenções direcionadas à promoção da saúde da mulher como um todo ${ }^{8}$.

O Ministério da Saúde ${ }^{8}$ recomenda iniciar acompanhamento da gestante no primeiro trimestre de gravidez e a realizar pelo menos seis consultas (sendo, no mínimo, duas realizadas por médico). Os principais procedimentos recomendados para as consultas são: exame físico (peso e estado nutricional da gestante; estatura; pulso e temperatura; pressão arterial; inspeção de pele, mucosas e tireóide; ausculta cárdio-pulmonar; exame de membros inferiores), exame ginecológico (exame de mamas, altura uterina, batimentos cárdio-fetais, palpação de gânglios e genitália, exame especular); exames laboratoriais de rotina (tipagem sangüínea, VDRL, urina e hemoglobina). Todas as gestantes devem receber, segundo estas normas, suplementação de ferro (independentemente do nível de hemoglobina) e orientação quanto ao aleitamento materno, entre outros procedimentos. Serão feitos exames de secreção vaginal, "preventivo de câncer de colo de útero" e vacina antitetânica apenas se houver indicação.

A mediana de consultas de pré-natal no Brasil em 1996 foi 7,4, variando de 6,3 na 
região Norte a 8,5 na região Sul. Nesta região, 6,3\% das mães não realizaram consultas de pré-natal ${ }^{9}$. Em todo o Brasil, apenas dois terços das gestantes iniciaram o prénatal até o terceiro mês de gestação, sendo que no Sul, este índice foi de $79,7 \%$ e, no Nordeste, $51,9 \%{ }^{9}$. Na coorte de Pelotas de 1982, o número de consultas de pré-natal foi inadequado para $5,5 \%$ das gestantes particulares, $22,9 \%$ das atendidas pelo sistema previdenciário e $77 \%$ entre mulheres sem filiação previdenciária (indigentes). Ao se avaliar conforme o critério de risco para morte perinatal e baixo peso ao nascer, o atendimento pré-natal foi inadequado em $31,7 \%$ das mulheres de alto risco e em $12,1 \%$ das mulheres de baixo risco ${ }^{7}$. Resultados semelhantes foram observados nesta mesma localidade 11 anos depois ${ }^{2}$. Estudo realizado na cidade de São Paulo em meados da década de 90 mostrou que, em média, as mulheres ralizaram 8,0 consultas durante toda a gestação, $88 \%$ fizeram cinco ou mais consultas e $69 \%$ iniciaram o pré-natal ainda no primeiro trimestre, $7 \%$ não fizeram uma única consulta de pré-natal e as mais pobres receberam o pior tipo de assistência ${ }^{4}$. Em São Luis, Maranhão, a cobertura do pré-natal foi de $89,5 \%$. Destas, $62,9 \%$ realizaram cinco ou mais consultas pré-natais e $60,2 \%$ iniciaram estas consultas ainda no primeiro trimestre. O número médio de consultas foi de 6,6 , e 9,3\% delas não tiveram qualquer tipo de atendimento ${ }^{3}$.

O presente estudo, realizado na zona urbana de Criciúma, Estado de Santa Catarina, teve por objetivo avaliar a atenção ao pré-natal, ao parto e ao recém-nascido segundo critérios socioeconômicos e procedimentos técnicos realizados.

\section{Metodologia}

O município de Criciúma está situado no Estado de Santa Catarina, Sul do Brasil, e em 1996 possuía 159.101 habitantes, $15 \%$ dos quais eram menores de seis anos ${ }^{10}$. A rede hospitalar municipal era composta de quatro hospitais, todos privados, sendo três contratados e um filantrópico, totalizando 814 leitos ${ }^{12}$. Havia 39 Unidades de Saúde, 17 das quais contavam com o Programa Saúde da Família (PSF), iniciado no final de 1994. Faziam parte também da rede municipal quatro unidades referenciais de saúde, duas unidades especializadas, um centro de saúde e seis unidades odontológicas móveis. Havia ainda no município o programa de atenção materno-infantil, de base comunitária, desenvolvido pela Pastoral da Criança, que cobria $4,8 \%$ das crianças menores de três $\operatorname{anos}^{12}$. Na ocasião, Criciúma estava entre os 30 melhores municípios do Estado de Santa Catarina e entre os 50 melhores do Brasil ${ }^{6}$.

O presente estudo, que se utiliza de um delineamento transversal com um forte componente retrospectivo, faz parte de um trabalho mais amplo ${ }^{12,13} \mathrm{e}$ foi realizado em 1996, na área urbana de Criciúma, sendo incluída a sede do município e seu único distrito, Rio Maina. Os 113 setores censitários disponíveis foram divididos em dois estratos: o primeiro, com forte presença da Pastoral da Criança, abrangia 37 setores, e o segundo, 76 setores. A amostra selecionada para o presente estudo foi composta de todos os 37 setores do primeiro estrato e 15 setores (20\%) do segundo estrato, selecionados através de amostragem proporcional ao tamanho. No primeiro estrato foram visitados $54 \%$ dos domicílios de cada setor e, no segundo estrato, foram visitados 155 domicílios em cada setor.

Ainda que em cada estrato houvesse igual chance de entrada no estudo para todas as crianças, houve proposital sobreamostragem do primeiro estrato. Isto foi compensado na análise, através de ponderação, tornando os resultados representativos para a totalidade da população urbana do município.

A coleta de dados foi realizada por 14 entrevistadoras com, no mínimo, dez anos de escolaridade. Estas foram selecionadas após um treinamento teórico-prático de 80 horas e um estudo piloto. Fizeram ainda parte da equipe três supervisores de campo, um supervisor de qualidade (responsável pelas re-visitas) e os dois coordenadores da pesquisa. A coleta de dados foi conduzida nos meses de março a junho de 1996. 
Para iniciar a coleta, foi sorteada uma quadra em cada setor escolhido. Nesta quadra sorteou-se uma esquina e, no sentido horário, foram visitados, consecutivamente, todos os domicílios inicialmente previstos para cada setor. Foi aplicado um questionário para todas as crianças residentes e com até três anos de idade. Caso o domicílio estivesse fechado, perguntava-se pelo menos a dois vizinhos, se havia alguma criança no domicílio. Caso houvesse, retornava-se em outro dia e horário a fim de entrevistar o responsável pela criança: se, após quatro visitas em dias e horas diferentes, o mesmo não fosse encontrado, considerava-se essa criança como perda.

Os dados foram coletados através de questionário pré-codificado aplicado no domicílio da mãe ou pessoa responsável pela criança menor de três anos. Este questionário era codificado no mesmo dia pelo entrevistador e revisado pela equipe central na mesma semana para, posteriormente, ser duplamente digitado.

Foram coletadas informações sobre as seguintes variáveis: assistência à gestação $\mathrm{e}$ ao parto (realização de consultas de pré-natal, mês de início e número total de consultas realizadas), procedimentos técnicos realizados, por médico ou enfermeira, durante as consultas, e exames clínicos e laboratoriais, orientação sobre amamentação e imunização antitetânica, e prescrição de ferro no período. Também foram coletadas informações sobre local do parto, permanência em alojamento conjunto, etc. A partir destas variáveis, diversos indicadores foram construídos como, por exemplo, número adequado de consultas de pré-natal. Isto foi calculado dividindo-se o número de crianças com pelo menos seis consultas de pré-natal pelo número total de crianças na amostra. Foram também coletadas informações sobre variáveis demográficas (sexo, cor da pele e idade da criança), socioeconômicas (renda familiar total no mês que antecedeu a entrevista, escolaridade da mãe em anos completos com aprovação), variáveis ambientais (tipo de moradia e aglomeração familiar) e, finalmente, antecedentes repro- dutivos maternos (número de filhos nascidos vivos e mortos e intervalo interpartal).

A fim de verificar a qualidade dos dados coletados, foram revisitados cerca de $10 \%$ dos domicílios, ocasião em que partes do questionário foram repetidas. A concordância entre as respostas obtidas na primeira visita feita pelo entrevistador e as respostas obtidas na re-visita por um dos coordenadores foi avaliada através do índice de kappa ${ }^{14}$.

A análise estatística bivariada incluiu o teste do qui-quadrado para as tabelas de contingência ${ }^{14}$. Nas tabelas $2 \times 2$ foi utilizada a correção de continuidade de Pearson ${ }^{14}$. Utilizou-se o teste exato de Fischer quando a freqüência esperada era inferior a cinco ${ }^{14}$. Os testes estatísticos foram realizados através do programa SPSS ${ }^{15}$.

\section{Resultados}

Foram visitados 9.152 domicílios e encontradas 2.208 crianças menores de três anos. No primeiro estrato, foram visitados 6.827 domicílios onde havia 1.791 crianças ( $81,1 \%$ do total de crianças) e, no segundo estrato, 2.325 domicílios e 417 crianças (18,9\%). Não foi possível obter informações sobre 63 crianças $(2,8 \%)$. A causa principal destas perdas foi a ausência do morador (37 casos). No primeiro estrato, as perdas e recusas foram menores do que no segundo estrato (2,1\% versus $5,7 \%$ - $\mathrm{p}<0,001)$. Dos 14 respondentes que não souberam relatar os procedimentos, $31,5 \%$ (4) pertenciam ao primeiro quartil, $3,9 \%$ (1) ao segundo quartil, $27,6 \%$ (4) ao terceiro quartil e $36,9 \%$ (5) ao quarto quartil $(\mathrm{P}=0.37)$.

Para efeitos deste estudo, foram excluídas 20 crianças por parto gemelar e nove por parto trigemelar por receberem cuidados diferenciados no período gestacional. Outras três crianças foram excluídas do presente estudo por terem nascido no domicílio. Noventa e cinco por cento destas informações foram prestadas pela mãe biológica da criança.

Cinqüenta e um por cento das crianças eram do sexo masculino $(51,0 \%)$ e encontravam-se uniformemente distribuídas quanto à idade; $88,8 \%$ eram de cor branca; a 
Tabela 1 - Análise não ajustada da qualidade técnica do pré-natal, conforme renda familiar total em quartis*. Criciúma, 1996.

Table 1 - Non-adjusted analysis of the technical quality of antenatal care, per total family income quartile*. Criciúma, 1996.

\begin{tabular}{|c|c|c|c|c|c|}
\hline \multirow[t]{2}{*}{ Atividades } & \multirow{2}{*}{$\begin{array}{l}\% \text { crianças } \\
\text { ( } \mathrm{n} \text { total) }\end{array}$} & \multicolumn{4}{|c|}{ Renda Familiar Total } \\
\hline & & $1^{\circ}$ quartil & $2^{\circ}$ quartil & $3^{\circ}$ quartil & $4^{\circ}$ quartil $(t)$ \\
\hline \multicolumn{6}{|c|}{ Pré-natal } \\
\hline Fizeram pré-natal & $(2118)$ & & & & $(p=0,006)$ \\
\hline & $96,6 \%$ & $95,2 \%$ & $95,7 \%$ & $98,1 \%$ & $97,5 \%$ \\
\hline Iniciaram no $1^{\circ}$ trimestre & $(2032)$ & & & & $(p<0,001)$ \\
\hline & $83,2 \%$ & $73,9 \%$ & $86,7 \%$ & $86,5 \%$ & $87,7 \%$ \\
\hline Foram a 6 ou + consultas & $(2032)$ & & & & $(p<0,001)$ \\
\hline & $77,6 \%$ & $66,7 \%$ & $80,0 \%$ & $81,7 \%$ & $84,4 \%$ \\
\hline Iniciaram no $1^{\circ}$ trimestre e foram & $(1690)$ & & & & $(p<0,001)$ \\
\hline a 6 ou + consultas & $92,3 \%$ & $88,3 \%$ & $91,9 \%$ & $92,8 \%$ & $96,1 \%$ \\
\hline Início e $n^{\circ}$ consultas adequado & $(2032)$ & & & & $(p<0,001)$ \\
\hline & $76,7 \%$ & $65,3 \%$ & $79,7 \%$ & $80,2 \%$ & $84,4 \%$ \\
\hline \multicolumn{6}{|c|}{ Conduta no pré-natal } \\
\hline Verificação da data última menstruação & $(2019)$ & & & & $(p<0,001)$ \\
\hline Determinação do peso & $\begin{array}{l}96,9 \% \\
(2030)\end{array}$ & $95,0 \%$ & $96,1 \%$ & $97,6 \%$ & $\begin{array}{c}99,3 \% \\
(p=0,2 \ddagger)\end{array}$ \\
\hline & $98,6 \%$ & $98,3 \%$ & $97,8 \%$ & $99,2 \%$ & $99,1 \%$ \\
\hline Medida da altura uterina & $(2029)$ & & & & $(p=0,4 \neq)$ \\
\hline \multirow{3}{*}{ Medida da pressão arterial } & $98,3 \%$ & $97,6 \%$ & $98,3 \%$ & $98,3 \%$ & $99,0 \%$ \\
\hline & $(2027)$ & & & & $(p=0,05 \ddagger)$ \\
\hline & $97,4 \%$ & $98,0 \%$ & $97,0 \%$ & $96,1 \%$ & $98,7 \%$ \\
\hline \multirow[t]{2}{*}{ Exame ginecológico especular } & $(2014)$ & & & & $(p<0,001)$ \\
\hline & $50,9 \%$ & $47,2 \%$ & $40,3 \%$ & $46,4 \%$ & $70,2 \%$ \\
\hline \multirow[t]{2}{*}{ Prescrição de ferro } & $(2020)$ & & & & $(p<0,001)$ \\
\hline & $58,7 \%$ & $63,8 \%$ & $60,5 \%$ & $57,0 \%$ & $52,6 \%$ \\
\hline \multirow[t]{2}{*}{ Orientação sobre amamentação } & $(2027)$ & & & & $(p<0,001)$ \\
\hline & $71,5 \%$ & $67,6 \%$ & $69,6 \%$ & $70,1 \%$ & $79,7 \%$ \\
\hline \multirow[t]{2}{*}{ Exame de sangue } & $(2030)$ & & & & $(p=0,3 \neq)$ \\
\hline & $93,0 \%$ & $91,8 \%$ & $94,1 \%$ & $92,3 \%$ & $94,1 \%$ \\
\hline Exame das mamas & (2029) & & & & $(p<0,001)$ \\
\hline \multirow{3}{*}{ Orientação sobre vacina anti-tetânica } & $50,6 \%$ & $38,5 \%$ & $41,7 \%$ & $52,2 \%$ & $71,9 \%$ \\
\hline & (2022) & & & & $(p=0,4 \ddagger)$ \\
\hline & $79,4 \%$ & $79,5 \%$ & $81,8 \%$ & $78,9 \%$ & $77,4 \%$ \\
\hline
\end{tabular}

* O primeiro quartil incluiu famílias com renda familiar total menor ou igual a $\mathrm{R} \$ 300$; segundo quartil: $\mathrm{R} \$ 301$ a $\mathrm{R} \$ 408$; terceiro quartil: $\mathrm{R} \$ 481$ a $\mathrm{R} \$ 800$; quarto quartil: renda maior que $\mathrm{R} \$ 800$.

* The first quartile includes families whose total family income is less or equal to $R \$ 300,00$; second quartile: $R \$ 301$ to $R \$ 408$; third quartile: $R \$ 481$ to $R \$ 800$; fourth quartile: income higher than $\mathrm{R} \$ 800$

$\dagger \mathrm{p}$ valor $=$ Teste para tendência linear em proporções de Mantel-Haenszel $/ \uparrow p$ value $=$ Mantel-Haenszel test for linear trend in proportions

$\neq p$ valor $=$ Teste do qui-quadrado de Pearson $/ \neq p$ value $=$ Pearson's chi-square test

renda mensal total de suas famílias atingiu a mediana de $\mathrm{R} \$ 478,00$; seus pais e mães completaram, respectivamente, 7,3 e 6,7 anos de estudo (DP 3,5 anos para ambos); 9,9\% das mães relataram não ter companheiro; a idade média das mães era 27,6 anos completos (DP 6,8 anos); $18,0 \%$ das crianças moravam em malocas, casas de tijolo sem revestimen- to ou de madeira irregular; a mediana de aglomeração era 2,5 moradores para cada peça utilizada para dormir. $38,5 \%$ das crianças eram primogênitas.

Para 2,2\% (47) não foi possível obter informações sobre a realização de consultas de pré-natal. 96,6\% das demais realizaram pelo menos uma consulta de pré-natal, sen- 
do que $83.1 \%$ delas iniciaram estas consultas durante o primeiro trimestre de gestação. Dentre estas mulheres, $77,5 \%$ fizeram seis ou mais consultas, sendo sete a mediana de consultas. A cobertura de pré-natal adequado alcançou 76,6\% das crianças.

Quatorze entrevistados (0,7\%) não souberam relatar os procedimentos realizados pelo menos uma vez durante as consultas de pré-natal; $98,6 \%$ das gestantes tiveram seu peso determinado; $98,3 \%$, a medida da altura uterina verificada; $97,4 \%$, a pressão arterial mensurada; a data da última menstruação foi verificada em 96,9\% dos casos; realizaram exame de sangue $93,0 \%$ das gestantes; receberam orientação sobre imunização antitetânica 79,3\%; sobre amamentação, $71,3 \%$. Houve prescrição de ferro para $58,7 \%$ delas. Exame ginecológico especular e de mamas foi realizado em $51,0 \%$ e $50,6 \%$ das gestantes, respectivamente.

Foram realizados, em média, no pré-natal 7,9 procedimentos de um total de 10 avaliados (DP 1,5). Dentre as mães que foram incluídas na análise, três souberam informar sete procedimentos e uma mãe informou apenas seis procedimentos, enquanto as demais mães informaram a realização de pelo menos oito dos dez procedimentos pesquisados. A forma de pagamento do pré-natal foi através do SUS para 1.533 (74,5\%) das gestantes de forma; particular para $359(17,4 \%)$; e outras formas de pagamento para $167(8,1 \%)$.

A Figura 1 mostra que houve maior participação do SUS no atendimento pré-natal entre as crianças nascidas nos últimos 11 meses $(\mathrm{P}=0,02)$ em relação às nascidas há 12 meses ou mais. $\mathrm{O}$ atendimento ao recémnascido (Parto/RN) foi realizado basicamente em dois hospitais. O pagamento do "Parto/RN" foi efetivado pelo SUS em $81,0 \%$ dos casos, particular em 14,9\% e, através de outras formas de pagamento em $4,1 \%$. Foram pagos pelo SUS 83,9\% dos atendimentos "Parto/RN” no hospital A e 81,3\% no hospital B. Estes diferiram significativamente dos outros hospitais, onde $69,5 \%$ dos atendimentos “Parto/RN" foram pagos pelo SUS $(\mathrm{p}<0,001)$. Somente $37(1,8 \%)$ das 2.180 crianças foram colocadas para mamar nos pri-

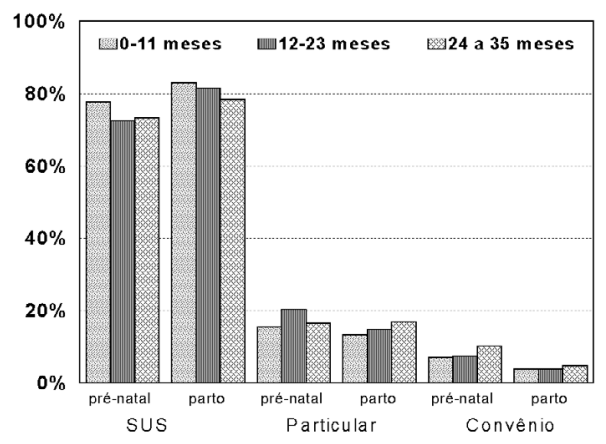

Figura 1 - Forma de pagamento do prénatal e parto, por idade da criança, no município de Criciúma, 1996.

Figure 1 - Form of payment of antenatal care and delivery according to age of child, in the city of Criciúma, 1996.

meiros 30 minutos do pós-parto; para 50,3\% das crianças o tempo foi superior a seis horas.

$\mathrm{O}$ alojamento conjunto integral foi oferecido a $35,0 \%$ das crianças. Houve associação significativa entre o hospital onde o parto foi realizado e a utilização do alojamento conjunto: No atendimento ao "Parto/RN" (Tabela 2) não foi encontrada diferença no tempo de colocação da criança para mamar após o parto. Este mesmo resultado foi encontrado quando esta análise foi refeita excluindo as 27 crianças que nunca mamaram. Esta exclusão foi testada em virtude das características especiais destas crianças, das quais destacamos o baixo peso ao nascer $(42,5 \%$ contra $5,6 \%$ das demais crianças $\mathrm{p}<0,001)$. As mães de menor renda utilizaram mais o alojamento conjunto integral e receberam menos orientação no hospital sobre como cuidar da criança e amamentação.

A Tabela 3 revela que as mães do quarto quartil de renda apresentaram média de procedimentos significativamente superiores aos demais quartis, tanto para os procedimentos de pré-natal como para os de atenção ao "Parto/RN". Na Tabela 4, verifica-se que as mães com duas ou três situações de "risco de saúde reprodutiva" apresentaram menor percentagem de atendimento com qualidade adequada (realização de no míni- 
Tabela 2 - Análise não ajustada da qualidade técnica do atendimento ao "Parto/RN", conforme quartis de renda familiar total*. Criciúma, 1996.

Table 2 - Non-adjusted analysis of the technical quality of delivery/neonatal care, per total family income quartile*. Criciúma, 1996.

\begin{tabular}{|c|c|c|c|c|c|}
\hline \multirow[t]{2}{*}{ Atividades } & \multirow{2}{*}{$\begin{array}{l}\% \text { crianças } \\
\text { (n total) }\end{array}$} & \multicolumn{4}{|c|}{ Renda Familiar Total } \\
\hline & & $1^{\circ}$ quartil & $2^{\circ}$ quartil & $3^{\circ}$ quartil & $4^{\circ}$ quartil $(\dagger)$ \\
\hline Colocada para mamar até 2 horas & (2034) & & & & $(p=0,2 \neq)$ \\
\hline após o nascimento & $11,5 \%$ & $13,4 \%$ & $11,3 \%$ & $9,5 \%$ & $11,6 \%$ \\
\hline Alojamento conjunto integral & $(2104)$ & & & & $(p=0,03)$ \\
\hline & $34,9 \%$ & $38,4 \%$ & $34,7 \%$ & $33,1 \%$ & $32,5 \%$ \\
\hline Orientação geral para a mãe no hospital & $(2087)$ & & & & $(p<0,001)$ \\
\hline & $44,3 \%$ & $37,6 \%$ & $43,1 \%$ & $43,0 \%$ & $55,6 \%$ \\
\hline Orientação sobre amamentação para a & (2087) & & & & $(p<0,001)$ \\
\hline mãe no hospital & $35,8 \%$ & $30,0 \%$ & $34,0 \%$ & $35,2 \%$ & $45,3 \%$ \\
\hline
\end{tabular}

* O primeiro quartil incluiu famílias com renda familiar total menor ou igual a $\mathrm{R} \$ 300$; segundo quartil: $\mathrm{R} \$ 301$ a $\mathrm{R} \$ 408$; terceiro quartil: $\mathrm{R} \$ 481$ a $\mathrm{R} \$ 800$; quarto quartil: renda maior que $\mathrm{R} \$ 800$.

* The first quartile includes families whose total family income is less or equal to $R \$ 300,00$; second quartile: $R \$ 301$ to $R \$ 408$; third quartile: $R \$ 481$ to $R \$ 800$; fourth quartile: income higher than $R \$ 800$

† $\mathrm{p}$ valor $=$ Teste para tendência linear em proporções de Mantel-Haenszel $/+p$ value $=$ Mantel-Haenszel test for linear trend in proportions

$\neq p$ valor $=$ Teste do qui-quadrado de Pearson $/ \neq p$ value $=$ Pearson's chi-square test

Tabela 3 - Análise não ajustada da média de procedimentos* de qualidade técnica do prénatal e atendimento ao "Parto/RN", conforme a renda familiar total. Criciúma, 1996.

Table 3 - Non-adjusted analysis of the average of procedures of technical quality of antenatal care and delivery/neonatal care, per total family income. Criciúma, 1996.

\begin{tabular}{|c|c|c|c|c|c|c|}
\hline \multirow[t]{2}{*}{ Serviço } & \multirow{2}{*}{$\begin{array}{l}\text { média geral } \\
\text { ( } \mathrm{n} \text { total) }\end{array}$} & \multirow[b]{2}{*}{$1^{\circ}$ quartil } & \multicolumn{3}{|c|}{ Renda Familiar Total* } & \multirow[t]{2}{*}{$p$ valor } \\
\hline & & & $2^{\circ}$ quartil & $3^{\circ}$ quartil & $4^{\circ}$ quartil & \\
\hline 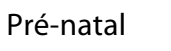 & $7,9(2031)$ & 7,8 & 7,7 & 7,9 & 8,4 & $<0,001 \neq$ \\
\hline “Parto/RN § & $0,90(2104)$ & 0,89 & 0,88 & 0,85 & 0,99 & $=0,02$ \\
\hline Total Q & 8,8 (2030) & 8,6 & 8,6 & 8,7 & 9,4 & $<0,001 \neq$ \\
\hline
\end{tabular}

* 10 procedimentos pesquisados: verificação da data última menstruação; Determinação do peso; Medida da altura uterina; Medida da pressão arterial; Exame ginecológico especular; Prescrição de ferro; Orientação sobre amamentação; Exame de sangue; Exame das mamas; Orientação sobre vacina anti-tetânica.

* 10 procedures studied: date of the last period; weight; uterine height; blood pressure; specular gynecological examination; iron prescription; breastfeeding orientation; blood test; breast examination; orientation on anti-tetanus vaccine.

† O primeiro quartil incluiu famílias com renda familiar total menor ou igual a $R \$ 300$; segundo quartil: $R \$ 301$ a $R \$ 408$; terceiro quartil: $\mathrm{R} \$ 481$ a $\mathrm{R} \$ 800$; quarto quartil: renda maior que $\mathrm{R} \$ 800$.

t The first quartile includes families whose total family income is less or equal to $R \$ 300,00$; second quartile: $R \$ 301$ to $R \$ 408$; third quartile: $R \$ 481$ to $R \$ 800$; fourth quartile: income higher than $R \$ 800$

‡ Análise de variância/‡ Variance analysis

$\S 3$ procedimentos pesquisados: criança colocada para mamar até 2 horas após o nascimento; Alojamento conjunto integral; Orientação para a mãe, no hospital. Excluídas 61 crianças devido ao fato de o entrevistado ignorar a resposta aos três procedimentos.

$\S 3$ procedures were studied: infant breastfed up to 2hours after birth; total room-in; orientation to the mother in the hospital. Sixty-one children were excluded because interviewee did not know the answer to the three procedures.

Q 13 procedimentos pesquisados: 10 procedimentos de Pré-natal e 3 de "Parto/RN" listados acima.

Q 13 procedures studied: 10 Antenatal procedures and 3 Delivery/Newborn procedures listed above

mo 11 procedimentos técnicos dos 13 previstos para o pré-natal e "Parto/RN"). A média de procedimentos realizados foi semelhante para os diversos grupos de risco. Apenas 17,5\% das crianças do hospital A permaneceram em alojamento conjunto em comparação aos $48,4 \%$ observados no hos- pital B e $57,5 \%$ nos demais.

No hospital, receberam orientação sobre como cuidar da criança $44,2 \%$ das mães. As principais orientações recebidas foram: amamentação (80,5\%); higiene (37,6\%); como cuidar do umbigo $(24,1 \%)$; imunização $(19,4 \%)$ e cólicas $(10,9 \%)$. 
Tabela 4 - Análise não ajustada comparando procedimentos de qualidade técnica do Pré-natal e atendimento ao "Parto/RN" conforme escore de risco referente a características de saúde reprodutiva. Criciúma, 1996.

Table 4 - Non-adjusted analysis comparing technical quality procedures in antenatal care and delivery/neonatal care, per risk score related to reproductive health features. Criciúma, 1996.

\begin{tabular}{|c|c|c|c|}
\hline Escore de risco* & No crianças (\%) & $\begin{array}{c}\% \text { qualidade atendimento } \\
\text { adequadat }(\mathrm{n})\end{array}$ & $\begin{array}{l}\text { Média de } \\
\text { procedimentos realizada }\end{array}$ \\
\hline 0 & 1307 (63,9\%) & $17,8 \%(233)$ & 8,8 \\
\hline 1 & $582(28,5 \%)$ & $22,1 \%(129)$ & 8,9 \\
\hline 2 ou 3 & $156(7,6 \%)$ & $12,8 \%(20) \neq$ & $8,7 \S$ \\
\hline \multicolumn{4}{|c|}{$\begin{array}{l}\text { * Considerou-se como antecedente de risco: idade, à época do parto, menor de } 18 \text { anos ou maior que } 34 \text { anos; ordem de nascimento maior que } 3 \text { filho; e } \\
\text { intervalo interpartal menor que } 24 \text { meses. } \\
\text { * Risk factors: age at time of delivery less than } 18 \text { or more than } 34 ; \text { birth order greater than third child; and inter-delivery interval of less than } 24 \text { months. } \\
+ \text { considerou-se como atendimento adequado a realização de, no mínimo, } 11 \text { procedimentos técnicos, dos quais } 10 \text { referentes ao Pré-natal e três ao "Parto/ } \\
\text { RN". } \\
\text { t adequate care was the performance of at least } 11 \text { technical procedures, } 10 \text { of which referring to antenatal care and three to delivery/neonatal care. } \\
\neq p \text { valor }=0,01 \text { para a categoria pelo teste do qui-quadrado de Pearson } \\
\neq p \text { value }=0.01 \text { for the category by Pearson's chi-square test. } \\
\S p \text { valor }=0,5 \text { para a categoria pela análise de variância } \\
\S p \text { value }=0.5 \text { for the category by variance analysis. }\end{array}$} \\
\hline
\end{tabular}

De forma semelhante ao que ocorreu no pré-natal, houve maior participação do SUS no atendimento ao "Parto/RN" no último ano ( $\mathrm{P}=0,04$ linear). Pode ser observado ainda, na Figura 1, que há maior número de atendimentos ao "Parto/RN" pelo SUS em relação ao pré-natal em todos os três anos pesquisados.

A Tabela 1 mostra as associações entre a qualidade técnica do atendimento de prénatal em relação aos quartis de renda familiar total. Observou-se que $95,2 \%$ das mães do primeiro quartil de renda familiar total realizaram o pré-natal, havendo tendência linear de maior prevalência de realização deste com o aumento da renda. As mães com menor renda iniciaram o pré-natal mais tarde e realizaram menos consultas. As mães com menor renda relataram com menos freqüência a verificação da data da última menstruação, a realização de exame ginecológico (especular e de mamas) e a orientação sobre amamentação. Estas mães referiram, no entanto, maior prescrição de ferro. Os índices kappa de concordância interobservadores, baseados na re-visita de cerca de $10 \%$ dos domicílios (237 crianças) foram: 0,82 para cor da criança (branca/parda/negra); 0,87 para consulta pré-natal (fez; não fez; ignorado); 0,79 para trimestre de início do pré-natal $\left(1^{\circ} ; 2^{\circ} ; 3^{\circ}\right)$; e 0,55 para o agrupamento de número de consultas de pré-natal adequado (menos que 6 ; 6 ou mais) e 0,43 para agrupamentos desta variável de dois em dois meses.

\section{Discussão}

O presente estudo revela a qualidade do pré-natal e do atendimento ao recém-nascido recebido por mães e filhos com até três anos de idade residente em área urbana do município de Criciúma, SC. Isto só foi possível devido à utilização de amostra probabilística e ao baixo percentual de perdas do estudo. As perdas nos estratos das amostras foram ligeiramente diferentes $(5,7 \%$ e $2,1 \%)$, o que não afeta os resultados apresentados.

Houve alto grau de concordância interobservadores (acima de 0,7), segundo a análise do índice kappa. O índice mais baixo observado deu-se na variável número de consultas de pré-natal (concordância razoável a boa). Uma vez que o número de consultas pode variar conforme o período da gestação, talvez este achado possa ser explicado pela dificuldade das mães em lembrar com exatidão o número de consultas. Há, neste ponto, alguma possibilidade de viés de recordatório, visto que algumas mães poderiam ter recebido um procedimento, mas esquecido de relatá-lo.Por 
exemplo, nos relatos de suplementação com ferro e de imunização contra tétano neonatal, não há diferença entre as classes e, no caso do ferro, o número de referências é maior para as pobres. Isto sugere que o viés de recordatório não é importante para explicar a falta de eqüidade. Um outro aspecto importante diz respeito ao tipo de delineamento utilizado. Nos estudos transversais, como o aqui utilizado, exposição e desfecho são medidos simultaneamente, o que impede determinar relações de causalidade. No entanto, as associações aqui estudadas já foram avaliadas através de estudos de coorte ${ }^{7,16}$. Assim, esta limitação parece não afetar as conclusões originárias deste estudo.

Como em outras localidades ${ }^{3,7}$, o SUS foi responsável pela imensa maioria dos atendimentos pré-natal e ao recém-nascido de Criciúma. A freqüência de atendimento ao "Parto/RN" pelo SUS $(81,0 \%)$ foi ainda maior que a de pré-natal $(74,5 \%)$. Isto poderia ser explicado pelo menor custo do pré-natal particular em relação ao parto, visto que além dos honorários médicos há despesas de hotelaria.

As mães de Criciúma apresentaram uma alta cobertura de pré-natal $(96,6 \%)$, semelhante à registrada na $\mathrm{PNDS}^{9}$ para a região Sul (94\%), cidade de Pelotas/RS, em 1997 $(95 \%)^{17}$, São Paulo, capital, em 1995-6 (93\%) , e Embu, SP, em $1996(96 \%)^{5}$, mas superior à taxa observada em São Luís, MA, em 1997-8 $(89 \%)^{3}$. A média de 7,0 consultas de pré-natal observada em Criciúma esteve abaixo das 8,3 consultas da PNDS $^{10}$, de Pelotas, RS $(8,2)^{17}$, São Paulo, capital $(8,0)$, mas acima do encontrado para São Luís, MA (6,6 consultas). Em Criciúma, o início do pré-natal ainda no primeiro trimestre foi superior ao observado para a região Sul $(79,7 \%)$, cidade de Pelotas $(71,9 \%)^{17}$, São Paulo, capital $(69 \%)^{4}$, Embu, SP $(72 \%)^{5}$, e São Luis $(63 \%)^{3}$. Os dados apresentados mostram que o município de Criciúma apresenta captação mais precoce das gestantes e menor número de consultas do que a média da região e também de vários outros municípios brasileiros ${ }^{3-5,7}$. No entanto, esta precocidade não se refletiu em maior concentração de consultas de pré- natal. O Programa de Assistência Integral à Saúde da Mulher do Ministério da Saúde (PAISM) considera que uma mulher é minimamente assistida no pré-natal quando comparece a um número de seis consultas durante a gravidez. Maior proporção de médicos por habitante também poderia facilitar ou induzir um maior número de consultas. Em Criciúma, havia um médico para cada 657 habitantes enquanto em Pelotas, por exemplo, esta proporção era de um médico para cada 260 habitantes (Conselhos Regionais de Medicina, comunicação pessoal).

Em Criciúma, três de cada quatro gestantes utilizaram o setor público para realizar o pré-natal, índice bastante semelhante ao encontrado em Pelotas e São Luis, onde este setor foi responsável por $78,5 \%$ e $84,2 \%$, respectivamente ${ }^{2,3}$. Ao analisar os procedimentos de pré-natal, tendo como parâmetro o preconizado pela Norma técnica do Ministério da Saúde ${ }^{8}$, verifica-se que tal como em Pelotas ${ }^{17}$, o serviço de pré-natal de Criciúma teve freqüência elevada de verificação da data da última menstruação (96,9\% para ambos os municípios), da medida da altura uterina (98,3\% e 99,0\% respectivamente), e de medida da pressão arterial $(97,4 \%$ e $99,5 \%)$. Boa parte destes procedimentos de pré-natal são desenvolvidos, na prática, por auxiliares de enfermagem. De certa forma, isto explica a semelhança encontrada em ambos os municípios: são procedimentos que se caracterizam por medidas de cunho individual, da prática cotidiana da enfermagem e bastante consolidadas na prestação de serviços básicos.

Encontrou-se freqüência aquém do esperado em orientação sobre a amamentação durante o pré-natal $(71,3 \%$ e $67,1 \%$ para Criciúma e Pelotas $\left.{ }^{17}\right)$. A freqüência de orientação sobre como cuidar da criança no hospital do parto foi ainda mais baixa: menos da metade das mães a recebeu. Estes procedimentos, em relação aos descritos no parágrafo anterior, pressupõem habilidades distintas e/ou participação de outros profissionais da equipe de saúde. Em Criciúma, os procedimentos que necessitam maior envolvimento médico (exames ginecológico especular e de mamas e prescrição de ferro) 
foram realizados com menos freqüência. Sendo norma do Ministério da Saúde ${ }^{8}$ a realização destes procedimentos como rotina para todas as gestantes, esperar-se-ia uma prevalência mais alta dos mesmos. Verificase em Criciúma menor prevalência de exame ginecológico especular (51,0\%), em comparação com Pelotas ${ }^{17}$ (73,2\%). Em relação ao exame das mamas as freqüências foram 50,6\% em Criciúma, $61,3 \%$ em Pelotas e $62,9 \%$ em Embu, respectivamente. A diferença encontrada entre estes municípios poderia ser explicada pela presença de outros serviços médicos, principalmente aqueles ligados a universidades, de onde se esperaria propedêutica melhor estabelecida.

A população de baixa renda apresentou menor probabilidade de receber atendimento pré-natal adequado, seja por apresentar maior freqüência de mães que não o fazem, seja por iniciar o pré-natal mais tardiamente, ou ainda por receber atendimento com menor qualidade técnica. Nas coortes de Pelotas de 1982 e 1993², na cidade de São Paulo ${ }^{4}$, São Luis $^{3}$ e Embu ${ }^{5}$, também se observou, no geral, que as mulheres de baixa renda foram proporcionalmente as que menos procuraram cuidados de pré-natal, tiveram menor média de consultas e buscaram assistência médica mais tardiamente. Isto talvez possa ser explicado por dificuldades de acesso, visto que os mais pobres esperam mais do que o dobro do tempo para serem atendidos ${ }^{18}$.

Entre os dez procedimentos de pré-natal pesquisados, apenas a prescrição de ferro foi significativamente maior entre os mais pobres e, ainda assim, apenas 1,2 vezes maior, considerando os quartis extremos. Dentre os demais procedimentos, destacamos os exames ginecológicos especular e de mamas. Nestes, a população do maior quartil de renda tem 1,5 e 1,9 vezes mais procedimentos, respectivamente, que o primeiro quartil. Estes procedimentos dependem de médico e exigem maior tempo de consulta. Este tempo não foi dedicado principalmente às mais pobres, talvez porque estas tendam a um menor poder de pressão sobre os serviços de saúde na reivindicação de atendimento de qualidade. $\mathrm{O}$ alojamento con- junto foi mais prevalente para a população de menor renda: foi de 1,2 vezes a diferença entre os quartis extremos. No entanto, as mães mais pobres foram as que menos receberam orientação sobre os cuidados com a criança e a amamentação (1,5 vezes menos que o último quartil, para ambas as orientações). As mães pobres são justamente as menos escolarizadas e com presumível menor acesso às informações necessárias.

Crianças de famílias com baixa renda são hospitalizadas com mais freqüência ${ }^{16}$. As crianças não amamentadas morrem mais por doenças infecciosas ${ }^{7}$ e são internadas 17 vezes mais por pneumonia do que as exclusivamente amamentadas ao seio materno ${ }^{19}$. Assim, é importante ressaltar que as práticas de aleitamento materno beneficiariam em especial as crianças cujas mães têm baixa escolaridade e vivem em situações de saneamento precário ${ }^{7}$, tendo sido justamente estas as que receberam menos orientação. A análise das médias de procedimentos realizados tanto no pré-natal como no "Parto/ RN" também evidencia a tendência de mães com menor renda receberem menor número de procedimentos. No entanto, há diferenças entre o atendimento ao pré-natal e ao "Parto/RN": enquanto no primeiro, em média, apenas um em cada cinco procedimentos não foi realizado, no atendimento ao "Parto/RN" deixaram de ser realizados dois dos três procedimentos. Esta diferença poderia ser explicada pelo fato de $\mathrm{o}$ atendimento ao "Parto/RN" ser único, enquanto o pré-natal é uma seqüência de atividades de controle com procedimentos semelhantes. Assim, os procedimentos "Parto/RN" seriam mais vulneráveis à não realização. Parte desta diferença poderia ainda ser explicada pelo fato de os procedimentos de atendimento pré-natal dependerem quase exclusivamente da capacitação do pessoal de enfermagem e do médico no atendimento individual, enquanto os procedimentos de "Parto/RN" dependem também da organização e da estrutura hospitalar.

As gestantes classificadas como de risco, segundo suas características de saúde reprodutiva, tampouco obtiveram a atenção 
especial necessária à sua situação, tendo recebido menos freqüentemente atendimento adequado. Usando critérios de risco diferentes do presente estudo, Dias-da-Costa ${ }^{2}$ descreveu que a média de consultas foi de 8,8 para as de gestantes de baixo risco e 6,8 para as de alto risco. Entre as primeiras, $4,9 \%$ fizeram a primeira consulta após o quinto mês, enquanto para as de alto risco, esta proporção foi de $13,9 \%$.

A Lei dos Cuidados Inversos descrita por Hart $(1971)^{1}$ foi encontrada em Criciúma, conforme exposto acima. Esta situação é compatível com diversos outros estudos brasileiros ${ }^{2-5,7,9,18,19}$ reforçando a idéia de que populações marginalizadas são as que menos recebem atendimento dos serviços de saúde. Assim, estes serviços ao "invés de corrigir, acentuam os diferenciais, através de uma discriminação contra as mães e crianças mais pobres”. Dentre todas as variáveis mensuradas de qualidade do serviço, o início precoce do pré-natal dependeria da disponibilidade da gestante em procurá-lo, da capacidade de oferta do serviço e ainda do acesso ao mesmo ${ }^{20}$. O número de consultas realizado está certamente associado à idade de início do pré-natal (quanto mais precoce mais consultas), mas também à capacidade do serviço de promover a adesão da gestan- te ao mesmo. Assim sendo, na análise da qualidade dos serviços, estas duas variáveis devem ser analisadas cautelosamente em virtude de poderem ser determinadas tanto pelos serviços como por motivos associados à gestante. No entanto, os procedimentos mensurados de qualidade técnica do prénatal deveriam ser efetuados já na primeira consulta, o que depende exclusivamente dos serviços. Este estudo mostrou que, na zona urbana de Criciúma, os mais pobres recebem atendimento de menor qualidade durante as consultas de pré-natal. Para contornar este quadro, seria necessário: a) utilizar indicadores de qualidade da atenção como o exame ginecológico e de mamas; $b$ ) buscar estratégias para captar mais precocemente as gestantes de menor renda; c) utilizar enfoque de risco: as mais pobres e gestantes com risco devem receber atenção diferenciada (discriminação positiva); d) retreinar os médicos quanto à importância de seus procedimentos de pré-natal, especialmente com relação ao exame ginecológico e de mamas; e) discussão destes resultados nos conselhos populares de saúde, conselho municipal de saúde e com os programas que mais intensamente atingem a população de menor poder aquisitivo, em especial o PSF, PACS e a Pastoral da Criança.

\section{Referências}

1. Hart JT. The inverse care law. Lancet 1971 (Feb); 40512.

2. Dias-da-Costa J, Victora CG, Barros FC, Halpern R, Horta BL, Manzolli P. Assistência médica maternoinfantil em duas coortes de base populacional no Sul do Brasil: tendências e diferenciais. Cad Saúde Pública 1996; 12 (S1): 59-66.

3. Coimbra LC, Silva AAM, Mochela EG, Alves TSSB, Ribeiro VS, Aragão VMF et al. Fatores associados à inadequação do uso da assistência pré-natal. Rev Saúde Pública 2003; 37(4): 456-62.

4. Monteiro CA, França Jr I, Conde WL. Evolução da assistência materno-infantil na Cidade de São Paulo (1984-1996). Rev Saúde Pública 2000, 34 (S6): 19-25.

5. Puccini RF, Pedroso GC, Silva EMK, Araújo NS, Silva NN. Eqüidade na atenção pré-natal e ao parto em área da Região Metropolitana de São Paulo, 1996. Cad Saúde Pública 2003; 19(1): 35-45.
6. UNICEF, IBGE. Municípios brasileiros: crianças e suas condições de sobrevivência. Censo Demográfico 1991. Fundo das Nações Unidas para a Infância - UNICEF e Fundação Instituto Brasileiro de Geografia e Estatística - FIGBE. Brasília; 1994.

7. Victora CG, Barros FC, Vaughan JP. Epidemiologia da desigualdade. 2a ed. São Paulo: Hucitec; 1989.

8. Ministério da Saúde. Assistência pré-natal. 2a ed. Brasília: Centro de Documentação do Ministério da Saúde; 1988.

9. Sociedade Civil Bem-Estar Familiar no Brasil (BENFAM). Pesquisa Nacional sobre Demografia e Saúde 1996. Relatório Preliminar. Rio de Janeiro: BENFAM; 1996.

10. IBGE (Instituto Brasileiro de Geografia e Estatística). Contagem da população, 1996. Rio de Janeiro: IBGE; 1997. 
11. Ministério da Saúde. DATASUS. Sistema de Informações Hospitalares do SUS (SIH/SUS). [banco de dados online]. Disponível em <URL:http:// www.datasus.gov.br> [2000 nov 11].

12. Neumann NA, Victora CG, Halpern R, Guimarães PRV, Cesar JA. A Pastoral da Criança em Criciúma, Santa Catarina, Brasil: cobertura e características sóciodemográficas das famílias participantes. Cad Saúde Pública 1999; 15(3): 543-52.

13. Neumann NA, Victora CG, Halpern R, Guimarães PRV, Cesar JA. Desempenho da Pastoral da Crianca na promoção de ações de sobrevivência infantil e na educação em saúde em Criciúma, uma cidade do sul do Brasil. Pam Am J Public Health 1999; 5(6).

14. Kirkwood BR. Essentials of medical statistics. London: Blackwell Science Ltd; 1999.

15. SPSS (Statistical Package for the Social Sciences). Release 6.1, standard version. Chicago: SPSS inc; 1994.
16. Cesar JA, Victora CG, Santos IS, Barros FC, Albernaz EP, Oliveira LM et al. Hospitalização por pneumonia: influência de fatores socioeconômicos e gestacionais em uma coorte de crianças no Sul do Brasil. Rev Saúde Pública 1997; 31(1): 53-61.

17. Santos IS, Baroni RC, Minotto I, Klumb AG. Critérios de escolha de postos de saúde para acompanhamento pré-natal em Pelotas (RS). Rev Saúde Pública 2000; 34: 603-9.

18. Travassos C, Viacava F, Fernandes C, Almeida CM. Desigualdades geográficas e sociais na utilização de serviços de saúde no Brasil. Ciência e Saúde Coletiva 2000; 5(1): 133-49.

19. Cesar JA, Victora CG, Barros FC, Santos IS, Flores JA. Impact of breast feeding on admission for pneumonia during postneonatal period in Brazil: nested casecontrol study. BMJ 1999; 318: 316-20.

20. Kaestner R. Health insurance, the quantity and quality of prenatal care, and infant health. Inquiry 1999; 36:162-175.

Recebido em: 10/02/2003 Versão final reapresentada em: 20/10/2003 Aprovação em: 30/10/2003 